\title{
Purification, crystallization and preliminary $X$-ray diffraction studies of retinal dehydrogenase type II
}

Audrey L. Lamb, ${ }^{a}$ Xianshu Wang, ${ }^{b}$ Joseph L. NAPOlI ${ }^{b}$ And Marcia E. Newcomer ${ }^{a} *$ at ${ }^{a}$ Department of Biochemistry, Vanderbilt University School of Medicine, Nashville, TN 37232, USA, and ${ }^{b}$ Department of Biochemistry, School of Medicine and Biomedical Sciences, State University of New York at Buffalo, Buffalo, NY 14214, USA. E-mail: newcomer@lhmrba.hh.vanderbilt.edu

(Received 5 August 1997; accepted 20 October 1997)

\begin{abstract}
One enzyme which catalyzes the last step of the formation of the hormone retinoic acid from vitamin A (retinol) is retinal dehydrogenase type II (RalDH2). RalDH2, expressed in the Escherichia coli BL21(DE3) strain, was purified and crystallized using ammonium sulfate as a precipitant. These crystals belong to the space group $P 2_{1} 2_{1} 2_{1}(a=108, b=150, c=168 \AA$, $\alpha=\beta=\gamma=90^{\circ}$ ).
\end{abstract}

\section{Introduction}

In vivo retinoic acid is produced from retinol in a two-step process which requires the action of binding proteins and enzymes: retinol is oxidized to retinal which is in turn oxidized to retinoic acid. Cellular retinol binding proteins (CRBP) and cellular retinoic acid binding proteins (CRABP) may protect the retinoids from inappropriate chemical modification, and may protect the cell from the amphipathic properties of the retinoid or the uncontrolled signaling effects of unchaperoned hormone (Napoli, 1996). Liganded binding proteins act as substrates for the enzymes that metabolize retinoids (Napoli et al., 1995; Boerman \& Napoli, 1995b; Ong et al., 1994). For example, retinol bound to CRBP acts as a substrate for retinol dehydrogenases which convert retinol to retinal (Napoli et al., 1995; Boerman \& Napoli, 1995a,b). It has been proposed that retinal bound to CRBP may act as the substrate for retinal dehydrogenase, which catalyzes the production of retinoic acid (Napoli, 1996). Retinoic acid then binds to CRABP for further metabolism by enzymes such as the P450's (Boylan \& Gudas, 1991), or is sequestered for nuclear localization and activation of the transcription factors RAR and RXR (Giguere, 1994). It has been proposed that retinoic acid may also be exported, affecting cells that cannot synthesize the hormone (Napoli, 1996).

RalDH2 belongs to the superfamily of aldehyde dehydrogenases which is subdivided into three classes. Classes I and II are comprised of tetrameric isozymes (subunit $\simeq 55 \mathrm{kDa}$ ). The isozymes of class I are cytosolic while those of class II are mitochondrial (Lindahl \& Hempel, 1991). Class III aldehyde dehydrogenases are smaller dimeric and primarily tumor specific, inducible or found in the endoplasmic reticulum (Hempel \& Lindahl, 1989). All three classes require the cofactor nicotinamide adenine dinucleotide. Classes I and II share approximately $70 \%$ sequence identity. However, within a class, these enzymes are approximately $95 \%$ identical (Hempel \& Lindahl, 1989). X-ray crystallographic structures of a class II aldehyde dehydrogenase and of a class III aldehyde dehydrogenase have been recently reported (Steinmetz et al., 1997; Liu et al., 1997).

RalDH2 is a homotetramer of $55 \mathrm{kDa}$ monomers which is approximately $70 \%$ identical to either the class I or class II enzymes (Zhao et al., 1996; Wang et al., 1996). RalDH2 catalyzes the formation of retinoic acid in mouse embryonic eye and trunk/spinal cord (Zhao et al., 1996), and rat testis (Wang et al., 1996). Zhao and colleagues have suggested that RalDH2 localization and the subsequent production of retinoic acid by the enzyme allows for nested gene activation and pattern formation in the early embryo (Zhao et al., 1996).

Based on the recently reported structures of the class II and class III enzymes, one would assume that a crystal structure of RalDH2 will display an $\alpha / \beta$ catalytic domain, a Rossmann fold nucleotide-binding domain, and a $\beta$-sheet tetramerization domain (Steinmetz et al., 1997; Liu et al., 1997). In order to understand better the mechanism of retinal specificity of this aldehyde dehydrogenase, we have initiated crystal structure determination studies. These and future studies may also elucidate the possible interaction domains of RalDH2 for other proteins in the enzymatic pathway, such as the cellular retinoid binding proteins.

\section{Materials and methods}

2.1. Expression and purification of rat retinal dehydrogenase type II

The plasmid containing the coding sequence of RalDH2 with an $\mathrm{N}$-terminal polyhistidine tag was transformed into BL21(DE3) E. coli by the $\mathrm{CaCl}_{2}$ method. Bacteria were grown in Terrific Broth (Difco) at $310 \mathrm{~K}$ until the absorbance at $A_{600}$ reached 0.6-0.8. The temperature was lowered to $291 \mathrm{~K}$ and the $E$. coli were induced with $0.4 \mathrm{~m} M$ isopropyl $\beta$-D-thiogalactopyranoside for $24 \mathrm{~h}$.

\subsection{Purification of RalDH2}

E. coli were pelleted $(5000 \mathrm{~g}, 277 \mathrm{~K}, 15 \mathrm{~min})$, resuspended in lysis buffer $(20 \mathrm{~m} M$ imidazole acetate $\mathrm{pH}$ 8.0, $2 \mathrm{~m} M \beta$ mercaptoethanol) with $2 \mathrm{~m} M$ PMSF, $20 \mu \mathrm{g} \mathrm{ml}^{-1}$ pepstatin, $20 \mu \mathrm{g} \mathrm{ml}^{-1}$ leupeptin and $200 \mathrm{U} \mathrm{ml}^{-1}$ DNase I, and lysed in a French press. The lysate was centrifuged $(10000 \mathrm{~g}, 277 \mathrm{~K}$, $30 \mathrm{~min}$ ), and the supernatant was loaded onto a $2 \mathrm{ml} \mathrm{Ni-NTA}$ agarose (Qiagen) batch column and incubated for $1 \mathrm{~h}$. The sample was allowed to flow through and the column was washed with lysis buffer until an $A_{280}$ less than 0.1 was obtained. The column was washed with wash buffer $[20 \mathrm{~m} M$ imidazole acetate $\mathrm{pH} 8.0,250 \mathrm{~m} M \mathrm{NaCl}, 2 \mathrm{~m} M \quad \beta$-mercaptoethanol, $1 \mathrm{~m} M \beta$-octylglucoside, $10 \%(v / v)$ glycerol] until the $A_{280}$ fell below 0.1. RalDH2 was eluted with a step gradient [20-220 $\mathrm{m} M$ imidazole acetate $\mathrm{pH} 8.0,250-300 \mathrm{~m} M \mathrm{NaCl}$, $2 \mathrm{~m} M \beta$-mercaptoethanol, $1 \mathrm{~m} M \beta$-octylglucoside, $10 \%(v / v)$ glycerol; $20 \mathrm{~m} M$ imidazole and $5 \mathrm{mM} \mathrm{NaCl}$ each step]. The fractions containing RalDH2 were concentrated under nitrogen in an Amicon stirred cell with a YM30 membrane and subsequently applied to an S300-sepharose column. The S300 
column was eluted with $20 \mathrm{~m} M$ Hepes $\mathrm{pH} 8.5,150 \mathrm{~m} M \mathrm{KCl}$, $1 \mathrm{~m} M$ EDTA, $2 \mathrm{~m} M \quad \beta$-mercaptoethanol. All buffers were thoroughly degassed with $\mathrm{He}$ sparging immediately prior to use.

An activity assay was conducted in $1 \mathrm{ml}$ of $20 \mathrm{~m} M$ Hepes $\mathrm{pH} 8.5,150 \mathrm{~m} M \mathrm{KCl}, 1 \mathrm{~m} M$ EDTA, $1 \mathrm{~m} M$ dithiothreitol, $2 \mathrm{~m} M$ NAD by monitoring the synthesis of NADH (increasing absorbance at $340 \mathrm{~nm}$ ) at $298 \mathrm{~K}$ for 2 min after a 1 min delay. Retinal was not used as a substrate because its $A_{340}$ completely conceals the absorbance of the synthesized NADH. The reaction was initiated by addition of the substrate analogs octanal $(5 \mathrm{~m} M)$ or decanal $(3 \mathrm{~m} M)$ in ethanol (Wang et al., 1996).

Thiol group determination was carried out as previously reported (Ellman, 1959).

\subsection{Crystallization}

Crystallization screens were initially carried out using the Hampton Research Crystal Screens 1 and 2 and the hangingdrop method. Drops were $2 \mu \mathrm{l}$ of the protein solution $\left(10 \mathrm{mg} \mathrm{ml}^{-1}\right)$ and an equal volume of the well solution. Screens were performed in the absence and presence of NAD, retinal

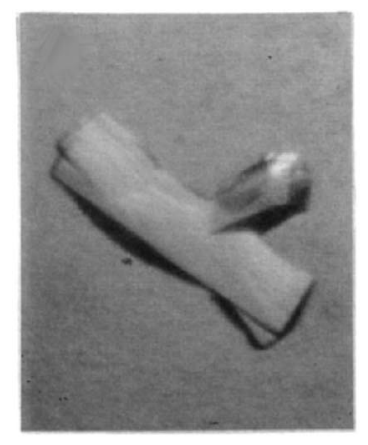

(a)

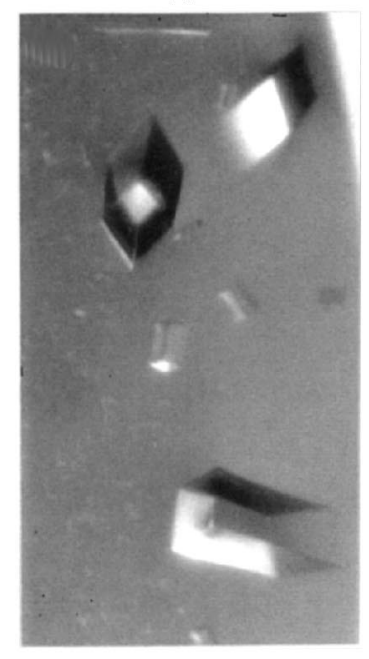

(c)
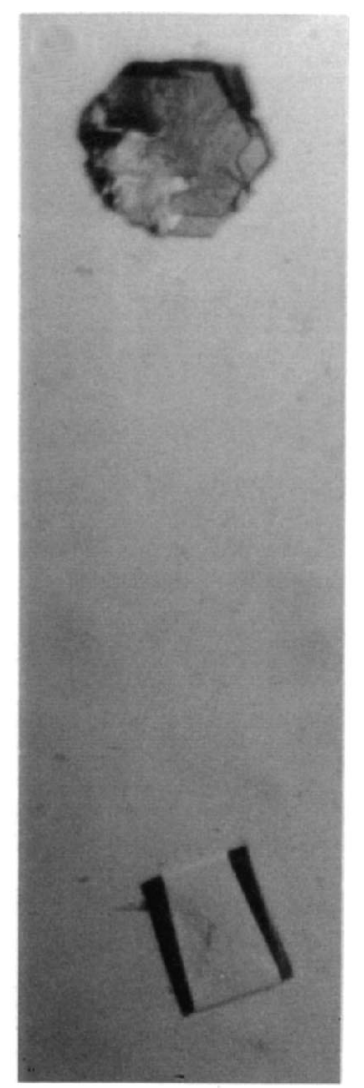

(b)
Fig. 1. (a) Type $A$ crystal grown after the first step of the purification (Ni-NTA agarose column). (b) On bottom, a type $A$ crystal grown after complete purification. On top, a cysteine crystal that grew simultaneously. (c) Type $B$ crystals. and/or 3-acetylpyridine adenine dinucleotide, a non-hydrolyzable NAD anaolgue. $A$-form crystals were grown by the sitting-drop method using a reservoir solution of $100 \mathrm{mM}$ imidazole acetate pH 6.5, 0.80-1.0 $\mathrm{M} \mathrm{NaOOCH}_{3}, 0.6 \mathrm{M} \mathrm{NaCl}$, 15-35 m $M$ cysteine, all thoroughly degassed with He sparging immediately prior to use. The trays were set up on ice with chilled buffers and then placed in a $298 \mathrm{~K}$ incubator. Drops of $10 \mu \mathrm{l}$ of protein solution $\left(5 \mathrm{mg} \mathrm{ml}^{-1}\right.$ with $2 \mathrm{mM}$ NAD) were mixed with an equal volume of the reservoir solution. $B$-form crystals were grown by the sitting-drop method using a reservoir solution of $100 \mathrm{mM}$ MES pH 6.5, 1.6-1.8 $M\left(\mathrm{NH}_{4}\right)_{2} \mathrm{SO}_{4}$, $5 \%$ dioxane, and $1-6 \%$ ethylene glycol, all thoroughly degassed with He sparging immediately prior to use. The trays were set up on ice with chilled buffers and then placed in a $298 \mathrm{~K}$ incubator. Drops of $10 \mu \mathrm{l}$ of protein solution $\left(5 \mathrm{mg} \mathrm{ml}^{-1}\right.$ with $2 \mathrm{~m} M \mathrm{NAD}, 2 \mathrm{~m} M \beta$-ionone) were mixed with an equal volume of the reservoir solution.

\subsection{Diffraction measurements}

Diffraction data of the $A$ crystals were collected at $123 \mathrm{~K}$ on an R-axis II image-plate system mounted on a Rigaku RU-200 rotating anode. The exposure time per frame $\left(0.5^{\circ}\right)$ was $20 \mathrm{~min}$ with a crystal-to-detector distance of $165 \mathrm{~mm}$. Diffraction data of the $B$ crystals were collected at $123 \mathrm{~K}$ at the Cornell High Energy Synchrotron Source at beamline F2. Oscillation frames of $0.7^{\circ}$ and $20 \mathrm{~s}$ were collected at $\lambda=1.07 \AA$ with a crystal-todetector distance of $80 \mathrm{~mm}$. A second data set of crystal $B$ diffraction data was collected at $123 \mathrm{~K}$ on an R-axis II imageplate system mounted on a Rigaku RU-200 rotating anode. The exposure time per frame $\left(0.7^{\circ}\right)$ was 45 min with a crystalto-detector distance of $165 \mathrm{~mm}$. All data were processed using DENZO (Otwinoswki, 1993; Minor, 1993).

\subsection{Diffraction analysis}

Pseudo precession photographs were generated using Precess (Furey \& Swaminthan, 1998). Self-rotation functions were calculated using $X$-PLOR (Brünger, 1992).

\section{Results and discussion}

RalDH2 which was eluted from the Ni-NTA agarose column was used to perform crystallization screens and was found to crystallize in the $A$ conditions, but the crystals were of poor quality (see Fig. 1a). Further purification was required for improved crystal quality. An S300 column eliminated contaminating bands noted on silver-stained polyacrylamide gel electrophoresis (PAGE) and resulted in a significant increase in the specific activity, as determined by the production of NADH in the presence of the substrate analog octanal. However, the increased purity was achieved at considerable cost to the yield of the preparation: approximately $20 \mathrm{mg} \mathrm{l}^{-1}$ culture after the Ni-NTA step to $5 \mathrm{mg} \mathrm{l}^{-1}$ culture after the S300 step.

Subsequently, it was noted that protein oxidation appeared to be another cause of poor crystal quality. Crystals grown without sparging the buffers with He did not crush easily as protein crystals should. In fact, these crystals could be dropped into deionized water and would not dissolve! This observation could be the result of free-thiol crosslinks as RalDH2 has at least three free thiols per monomer as determined by quantitation with $5,5^{\prime}$ dithiobis(2-nitrobenzoic acid). When the 
buffers are thoroughly degassed, the crystals grow to a suitable size for diffraction experiments and crush nicely as one would expect. Interestingly, protein not run on the S300 can grow crystals without cysteine as an additive. However, RalDH2 run over the S300 requires cysteine in the precipitating solution. The best RalDH2 $A$-form crystals are those that have undergone the complete purification procedure and are grown in the

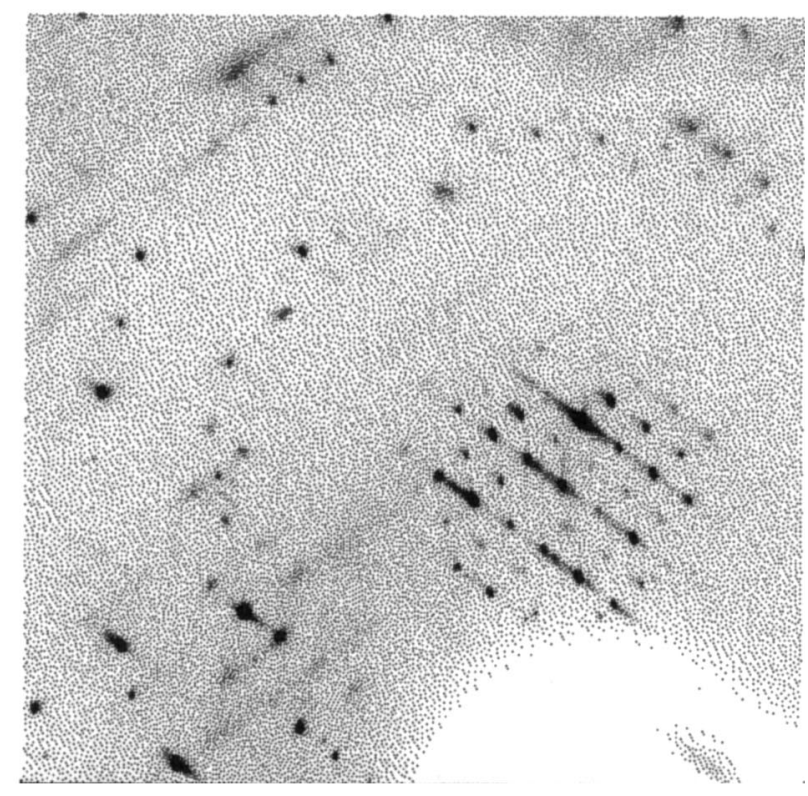

(a)

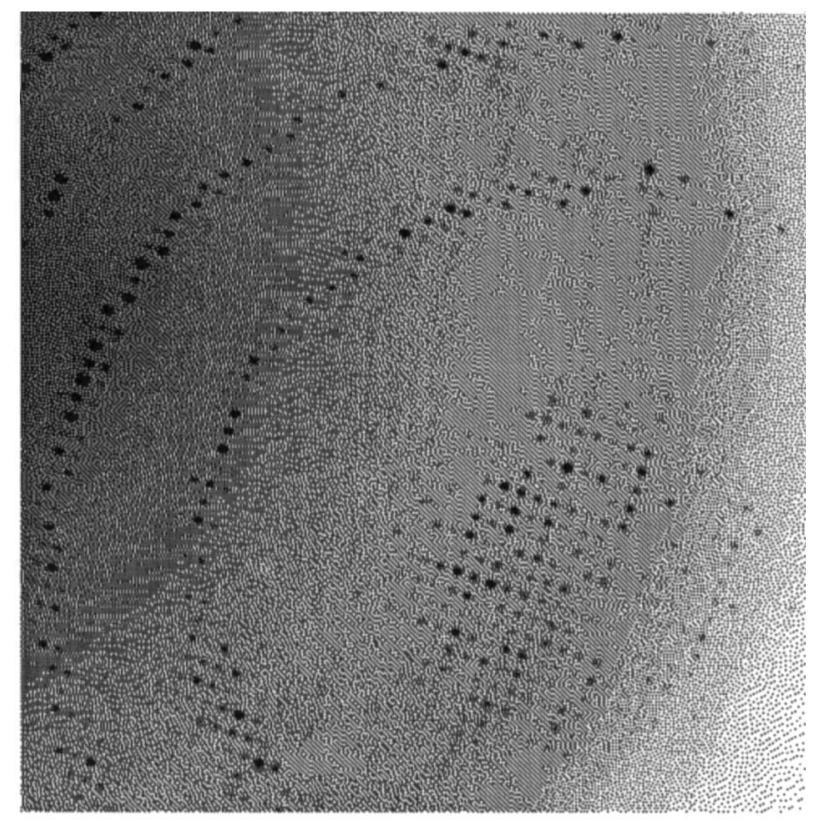

(b)

Fig. 2. (a) Diffraction pattern of type $A$ crystal; limit of the resolution of the image is $6 \AA$. (b) Diffraction pattern of type $B$ crystal; limit of the resolution of the image is $2.5 \mathrm{~A}$. presence of NAD. Initial $A$ crystals could be seen after $3 \mathrm{~d}$ and the crystals grew to $0.1 \times 0.1 \times 0.25 \mathrm{~mm}$ in $1-2$ weeks (see Fig. $1 b$ ). It should be noted that cysteine crystals grow simultaneously in the drop. These crystals can be differentiated from RalDH 2 crystals by their hexagonal morphology and because they also grow in the absence of protein (see Fig. $1 b$ ).

A single $A$-form RalDH2 crystal was used to collect a data set to $2.7 \AA\left(92.2 \%\right.$ complete, $\left.R_{\text {merge }}=8.8 \%\right)$. The data were processed in the space group $P 2$ with cell dimensions of $a=85$, $b=150, c=110 \AA, \beta=110^{\circ}$. If a tetramer comprises the asymmetric unit $\left(V=660000 \AA^{3}\right)$, the $V_{m}$ value is $2.97 \AA^{3} \mathrm{Da}^{-1}$ and the probable solvent content is $59 \%$ (Matthews, 1968). However, when examining data from $A$ form crystals and the fit generated by $D E N Z O$, one notes at least 100 extra spots per frame. Also, these crystals freeze very inconsistently and produce diffraction data with variable, and mostly unusable, mosaicity and $R_{\text {merge }}$ values. There was considerable streaking of the spots along the $a$ axis, frequently causing a doubling of this axis length during processing (see Fig. 2a). Many cryo-protectants were tried including sucrose, glucose, glycerol, ethylene glycol, PEG 400, and combinations of these.

In an attempt to find crystals which could be reproducibly frozen to yield high-quality X-ray data, a new crystallization screen was performed with the now well purified protein. The best crystals were grown with ammonium sulfate and dioxane as precipitants (the $B$ conditions) and in the presence of NAD and $\beta$-ionone. It was observed that nucleation was reduced in the presence of ethylene glycol. These crystals grow to $0.125 \times$ $0.125 \times 0.375 \mathrm{~mm}$ in $1-2$ weeks (see Fig. $1 c$ ), and freeze beautifully after soaking in reservoir solution with increasing amounts of ethylene glycol to a final concentration of $15 \%$. Data to $2.5 \AA$ were collected at the Cornell High Energy Synchrotron Source $(80 \%$ complete to $3.1 \AA$, $64 \%$ complete to $2.5 \AA, R_{\text {merge }}=7.7 \%$; see Fig. $2 b$ ). The crystals are orthorhombic and the data were processed in the space group $P 222$ with a unit cell of $a=108, b=150, c=168 \AA, \alpha=\beta=\gamma=90^{\circ}$. If a tetramer comprises the asymmetric unit $\left(V=680400 \AA^{3}\right)$, the $V_{m}$ value is $3.09 \AA^{3} \mathrm{Da}^{-1}$ and the solvent content is $60 \%$ (Matthews, 1968). Pseudo-precession images indicated the possibility of systematic absences of $0 k 0=2 n$ and $00 l=2 n$ but the $h 00$ information was unfortunately missing in the synchrotron data. A second data set collected on a conventional X-ray source was $100 \%$ complete but only diffracted to $3.2 \AA$ and with an $R_{\text {merge }}$ of $10.4 \%$. This data was useful in defining the space group as $P 2_{1} 2_{1} 2_{1}$ using pseudo-precession pictures (Furey \& Swaminthan, 1998).

A self-rotation function was calculated for the $B$ crystal form with data between 8.0 and $4.0 \AA$ using the program package $X$-PLOR (Brünger, 1992). Only peaks which aligned with space-group elements were observed. Therefore, it may be inferred that the twofold axes of the tetramer lie parallel to the twofold screw axes of the space group.

Molecular-replacement calculations of the $B$ crystal data using the recent class II aldehyde dehydrogenase structure (Steinmetz et al., 1997) as a search model are currently under way, and heavy-atom derivatives are being scanned.

The authors gratefully acknowledge support for this work from the National Institutes of Health grants AG13566 (to 
JLN), GM55420 (to MEN), and T32 GM08320 (support for ALL).

\section{References}

Boerman, M. H. E. M. \& Napoli, J. L. (1995a). Biochemistry, 34, 70277037.

Boerman, M. H. E. M. \& Napoli, J. L. (1995b). Arch. Biochem Biophys. 321, 434-441.

Boylan, J. F. \& Gudas, L. J. (1991). J. Cell Biol. 112, 965-972.

Brünger, A. T. (1992). X-PLOR: A system for X-ray crystallography and NMR. New Haven and London: Yale University Press.

Ellman, G. L. (1959). Arch. Biochem. Biophys. 82, 70-77.

Furey, W. \& Swaminthan, S. (1998). Methods Enzymol. 227, 590-620. Giguere, V. (1994). Endocrinol. Rev. 15, 61-79.

Hempel, J. \& Lindahl, R. (1989). Class III aldehyde dehydrogenase form rat liver: super family relationship to classes I and II and functional interpretation, edited by H. Weiner \& T. G. Flynn, pp. $3-$ 17. New York: Alan R. Liss.

Lindahl, R. \& Hempel, J. (1991). Aldehyde dehydrogenase: What Can Be Learned From a Baker's Dozen Sequences? edited by H. Weiner, B. Wermuth \& D. W. Crabb, pp. 1-8. New York: Plenum Press.
Liu, Z.-J., Sun, Y.-J., Rose, J., Chung, Y.-J., Hsaio, C.-D., Chang, W.-R., Kuo, I., Perozich, J., Lindahl, R., Hempel, J. \& Wang, B.-C. (1997). Nature Struct. Biol. 4, 317-326.

Matthews, B. M. (1968). J. Mol. Biol. 33, 491-497.

Minor, W. (1993). XDISPLAYF Program. Purdue University, West Lafeyette, Indiana, USA.

Napoli, J. L. (1996). FASEB J. 10, 993-1001.

Napoli, J. L., Boerman, M. H. E. M., Chai, X., Zhai, Y. \& Fiorella, P. D. (1995). J. Steroid Biochem. 53, 497-502.

Ong, D. E., Newcomer, M. E. \& Chytil, F. (1994). Cellular retinoid binding proteins, edited by M. B. Sporn, A. B. Roberts \& D. S. Goodman, pp. 283-317. New York: Raven Press Ltd.

Otwinoswki, Z. (1993). Oscillation data reduction program, Proceedings of the CCP4 study weekend, Data collection and processing, edited by L. Sawyer, N. Isaacs \& S. Bailey, pp. 56-62. Warrington: Daresbury Laboratory.

Steinmetz, C. G., Xie, P., Weiner, H. \& Hurley, T. D. (1997). Structure, 5, 701-711.

Wang, X., Penzes, P. \& Napoli, J. L. (1996). J. Biol. Chem. 271, 1628816293.

Zhao, D., McCaffery, P., Ivins, K. J., Neve, R. L., Hogan, P., Chin, W. W. \& Drager, U. C. (1996). Eur. J. Biochem. 240, 15-22. 